\title{
Glycan-mediated Functional Assembly of IL-1RI: Structural Insights into Completion of the Current Description for Immune Response
}

\author{
Maryam Azimzadeh Irani $^{1}$ and Mohammad Reza Ejtehadi ${ }^{2,3}$ \\ ${ }^{1}$ Shahid Beheshti University \\ ${ }^{2}$ Sharif University of Technology (SUT) \\ ${ }^{3}$ Sharif University of Technology
}

April 27, 2020

\begin{abstract}
Interleukin 1 receptor type I (IL-1RI) is a transmembrane receptor that triggers the inflammatory response. Understanding its detailed mechanism of action is crucial for treating immune disorders. IL-1RI is activated upon the formation of its "functional assembly" that occurs by binding of the cytokine and the accessory protein (IL-1RAcP) to the receptor. Glycosylation of the IL-1RI and IL-1RAcP play critical roles in the activation process. Here, extensive classical and accelerated molecular dynamics simulations are carried out to examine the structural role of glycosylation in the arrangement of the functional assembly at the atomic level. It is shown that the assembly is built in two types of non-signalling and signalling forms with the latter being the most frequently occurring form. The non-signalling assembly is formed by binding of the compact conformation of the glycosylated IL-1RI to the IL-1RAcP. In this type of assembly, the IL-1R is not accessible to the cytokines and thus it is unable to send signals to the cell. The signalling assembly is formed by binding of the extended glycosylated IL-1RI to the accessory protein. This assembly is locked in the extended form by persistent hydrogen bonds within and between the interconnected glycans of IL-1RI and IL-1RAcP. Cytokine binding site of the IL-1RI extracellular domain is exposed in this type of assembly and the signals could be transported to the cell. Moreover, contrary to the experimental interpretations, in both types of the assembly, binding of the IL-1RAcP to IL-1RI is primary to the cytokine binding to the complex.
\end{abstract}

\section{Introduction}

Interleukin 1 (IL-1) family of cytokines are key molecules in triggering the innate and acquired immune response upon injury, stress and more severe conditions such as cancer and asthma [1-3]. IL-1 cytokines share a similar 3D fold consisting of 12 anti-parallel $\beta$-strands that are connected by loops $[4,5]$. The similarity of their fold, rationalize the structural resemblance of their corresponding receptors. Signal transduction of the IL- 1 family members to the cytosol is carried out by specific transmembrane receptors including IL-1 Receptor type I (IL-1RI) [6, 7]. Due to the significant role of the IL-1 cytokines in establishing the immune response, several experimental [8-14] and computational $[6,15-17]$ studies have been carried out to explain the detailed functioning of these proteins. The most studied role of IL-1 cytokines is in activation of the IL1RI signalling complex that is triggered by agonist IL- $1 \alpha / \mathrm{IL}-1 \beta$ cytokine binding to the IL-1RI extracellular domain [14]. IL-1RI consists of an extracellular domain which itself consists of three immunoglobulin-like subdomains (DI-DIII), a transmembrane alpha-helix and an intracellular TIR domain (Figure 1.A) [18, 19]. The cytokine binding site is located at DI and DIII of the IL-1RI extracellular domain (Figure 1.C) $[4,20]$. X-ray crystal structures of the IL-1RI describe its activation mechanism in two steps. First is binding of IL-1 $\beta$ to IL-1RI through DI and DIII of the extracellular domain that forms the ligand-receptor complex. Second is binding of the IL-1 Accessory Protein (IL-1RAcP) to the above complex that forms 
the IL-1RI functional assembly $[14,20,21]$. It was suggested that IL-1 $\beta$ binding to IL-1RI extracellular domain leads to conformational changes required for binding of IL-1RAcP to the complex [13]. As the IL-1 $\beta$ binding was assumed to be the prerequisite of IL-1RAcP binding, the "IL-1RI-IL-1 $\beta$ " and "IL-1RI-IL$1 \beta$-IL-1RAcP" have been considered as "secondary" and "ternary" complex respectively [1]. However, in the above-mentioned activation mechanism, the role of IL-1RI glycosylation was not considered. Glycosylation is a post-translational modification under which the carbohydrate building blocks are bound to specific amino acids of the proteins [22-24]. Two major types of protein glycosylation occur inside the cell including N-linked and O-linked glycosylation [22]. N-linked glycosylation is known to be the primary type of glycosylation that modifies the proteins [25]. It often occurs at the Nitrogen atoms of Asparagines located within the specific Asn-X-Ser/Thr sequence [26]. Glycosylation has shown to be crucial for protein folding, stability and ligand binding [27-32]. More specifically, the significant role of glycosylation in functioning of other transmembrane receptors with similar activation mechanism to IL1RI has been comprehensively studied in recent years [3337] . Also, there are certain shreds of evidence that glycosylation could play important roles in cytokines and IL-1RAcP binding to IL-1RI [4, 13, 20]. However, those hints have remained unexplained. It was recently shown in a computational study, that the attachment of oligosaccharide glycans to the extracellular domain of IL-1RI shifts its dynamical pattern drastically from the common conformation that is obtained in X-ray crystallography [6]. The common conformation of the IL-1RI extracellular domain that is observed in most of X-ray crystal structures is the extended form [14]. In which the extracellular domain stands tall on the membrane and the cytokine binding cavity is exposed (Figure 1.C). In addition, among all crystal structures of IL-1RI, another type of rotated/compact conformation was also reported for IL-1RI extracellular domain in complex with a therapeutic peptide [21]. This conformation is adopted by the movement of DI and DIII of the extracellular domain towards each other that leads to occlusion of the cytokine binding site (Figure 1.A). Molecular Dynamics (MD) simulations of the extended IL-1RI in the glycosylated and unglycosylated forms showed that N-glycosylation often shifts the conformation towards the compact structure and maintains the receptor in that state [6]. As the compact conformation is not accessible to the cytokines, it was suggested that glycosylation does not favor cytokine binding to IL-1RI and acts as a regulatory mechanism [6]. However, this mechanism was introduced by solely considering the apo glycosylated IL-1RI and neglecting the role of IL-1RAcP as the other constructing unit of the functional assembly. The structural role of IL-1RAcP glycosylation in its dynamics and interactions with the glycosylated IL-1RI is also another crucial element [13] that has remained completely ambiguous at the atomic level. With the current information on the dynamics of the glycosylated apo IL-1RI that adopts the compact conformation [6], it seems implausible for IL- $1 \beta$ to bind to its compact receptor and form the secondary IL-1RI-IL- $1 \beta$ complex. Which could then interact with IL-1RAcP to construct the ternary IL-1RI-IL-1 $\beta$-IL-1RAcP complex that would be the functional assembly. This raises a very challenging yet fundamental question about the activation mechanism of the IL-1 signalling complex that could influence all aspects of the IL-1 related immune responses including computer-aided drug design and rational drug discovery studies.

Here, molecular modeling approaches were used to address the entire structural mechanism of the IL-1RI activation in the glycosylated form. The dynamical pattern of the fully glycosylated IL-RAcP was investigated to clarify if the accessory protein adopts a compact conformation similar to IL-1RI in the glycosylated form. It was shown that the fully glycosylated IL-1RAcP remains consistently extended in its dynamical pattern. Furthermore, dynamics of the glycosylated IL-1RI compact conformation in complex with the glycosylated IL-1RAcP was investigated using both conventional and advanced sampling techniques. The collected observations showed that although the interactions between the two proteins are weakened, the resulting complex is abiding. Suggesting that the IL-1RI-IL-1RAcP complex formation does not require the primary binding of IL- $1 \beta$ to IL-1RI and could be the secondary complex and not the ternary complex in the IL-1RI functional assembly (Figure 1.A-B). Although this mechanism is proposed solely by performing extended atomistic simulations of the complex, its very basic foundation is already experimentally validated. Adoption of the IL-1RI compact conformation in complex with a therapeutic peptide was shown in an X-ray crystal structure earlier [21]. This conformational arrangement was suggested to act as a down-regulation mechanism for IL-1RI functioning [21]. Dynamics of the glycosylated extended IL-1RI in complex with glycosylated IL-1RAcP was also studied and it was shown that the glycosylated "extended IL-1RI-IL-1RAcP" 
is the most stable form of the complex in which the IL-1RI is locked in the extended state by the glycans attached to its extracellular domain. In addition, IL- $1 \beta$ binding site is entirely exposed in this form. Hence, the IL-1RI-IL-1RAcP-IL-1 $\beta$ could be the ternary complex and the final step to form the functional assembly (Figure 1.C-E). The critical role of glycans in locking the extended IL-1RI and IL-1RAcP together was then explored by removing those glycans that result in either adoption of the compact conformation in IL-1RI and weakening of the complex, or in a close-by contact between the two proteins that leads to destabilizing fluctuations. For the rest of the text and figures, the glycosylated forms of IL-1RI and IL-1RAcP extracellular domains will be referred to as: gIL-1RI and gIL-1RAcP respectively.

\section{Methods}

\section{Model building of the glycosylated IL-1RAcP and two glycosylated complexes of the compact IL-1RI-IL-1RAcP and extended IL-1RI-IL-1RAcP}

Chain B of the PDB ID 3O4O [13] was selected as the initial model of the IL-1RAcP extracellular domain. For the fully glycosylated model, the structure was glycosylated at Asn37, Asn87, Asn176, Asn198 and Asn279 using the GLYCAM online builder [38]. An identical common type of eukaryotic oligosaccharide glycan was attached to all the glycosylation sites [22]. This oligosaccharide model is selected as a primary model of glycosylation that occurs in normal human cells [22]. Details of the structure and glycosidic linkages of the attached oligosaccharides can be found in Supplementary Figure 1. The same type of oligosaccharide was attached to Asn83, Asn176, Asn216, Asn232, Asn246 and Asn280 of the compact and extended IL1RI extracellular domain using the GLYCAM builder [38]. Glycosylated model of the compact IL-1RI-IL1RAcP complex was built by superimposing the compact conformation of IL-1RI onto the crystal structure of the extended complex (PDB ID 4DEP) [14]. This superimposition is reliable as the structure of the compact glycosylated IL-1RI was generated after several cycles of minimizations, heat-ups, equilibration and production MD that was performed on the glycosylated IL-1RI crystal structure (PDB ID 4DEP, chain E) [6]. The resulting complex was also minimized in two cycles of 7500 steps in total (see the molecular dynamics simulations section) to remove the plausible steric clashes within the complex. Extended glycosylated model of IL-1RI-IL-1RAcP complex was obtained by superimposing the IL-1RAcP structure to the crystal structure of the extended complex (PDB ID 4DEP). The resulting complex was minimized with a similar protocol mentioned above for the compact complex. Models for the control simulations of the extended IL-1RI-IL1RAcP complex with the removed glycans were built by not attaching the oligosaccharides to Asn216-IL1RI/Asn279-IL-1RAcP and Asn216, Asn232-IL-1RI/Asn279-IL-1RAcP of the complex and similar energy minimization protocol as mentioned above was applied to each model.

\section{Molecular dynamics simulations}

All molecular dynamics simulations were carried out with AMBER16 package [39, 40]. Parameters and topology files required for molecular dynamics were created with xleap module of AMBER package. Protein residues of IL-1RI and IL-1RAcP were presented with AMBER ff14SB force field [41]. Glycan units of the oligosaccharides attached to the IL-1RI and IL-1RAcP extracellular domains were presented with GLYCAM$06 \mathrm{j}$ force field [42]. Glycosylated IL-1RAcP and the fully and partially glycosylated complexes of compact IL-1RI-IL-1RAcP and extended IL-1RI-IL-1RAcP were immersed in a box of TIP3P waters keeping at least $10 \AA$ distance from the solute atoms to the edge of the box and the systems were neutralized by adding counter ions. All systems were minimized in two cycles of 1500 and 6000 steps of steepest descent/conjugate gradient [43]. The protein was restrained in the first cycle of each system to relax the waters and ions. The whole complex was then minimized in the second cycle with no restraint. Next, the systems were heated up to $300 \mathrm{~K}$ and equilibrated for $250 \mathrm{ps}$. Production runs were carried out for $50 \mathrm{~ns}$ in three/six replicates under isothermal-isobaric (NPT) ensemble scheme with the total simulation time of 1.2 $\mu \mathrm{s}$. A detailed description of all simulations can be found in Table S.1. Constant pressure dynamics was carried out by using a weak coupling algorithm [44] with the reference pressure set to 1 bar and maintained with a relaxation time of 1 ps. The Temperature was maintained at $300 \mathrm{~K}$ using Langevin dynamics $[45,46]$ with a collision frequency of $1 \mathrm{ps}-1 \mathrm{~K}$. The nonbonded interactions (electrostatic and VDW) were computed with a $9 \AA$ cut-off value and the particle mesh Ewald method was applied to simulate the long-range electrostatic interactions [34]. accelerated 
Molecular Dynamics (aMD) has shown to improve the insufficient phase-space sampling experienced in the classical MD significantly [47-49]. Thus, three replicates of 50ns aMD simulations were carried out on the fully glycosylated compact IL-1RI-IL-1RAcP to explore the stability of this complex. The average total and dihedral energies obtained from a 50ns classical MD of the fully glycosylated compact IL-1RI-IL-1RAcP complex were used for calculations of the boost energy (E) and acceleration parameter $(\alpha)$ for the aMD simulations. A dual boost in the total and torsional energy has been added to the system using the well established protocol that is developed by the team of Andrew McCammon [47-51] and has been integrated within the AMBER package [39]. Starting structure and the rest of the input parameters were the same as the ones explained for classical MD. All aMD simulations were followed by another 50ns classical MD to allow the system to regain its conventional dynamical pattern.

\section{Analyses of the MD trajectories}

RMSD and domain-domain/interacting amino acid pairs distances were calculated with VMD package [52] and cpptraj module of AMBER17 tools [53] respectively. All calculations were carried out over the 50ns of the simulations on the backbone atoms $(\mathrm{C} \alpha, \mathrm{C}$, and $\mathrm{N})$ of the protein complex. The trajectories were fitted to the initial model of each system in order to present the conformational changes of all simulations from a fixed reference point. Hydrogen bonds analysis was performed on the last 10ns of the simulations with the python code HBonanza_1.01 [54] and the bonds with persistently of $>75 \%$ are reported. All figures were made with VMD [52] and PyMOL [55].

\section{Results}

\section{Dynamics of the glycosylated IL-1RAcP}

Functional assembly of the IL-1RI is constructed by two glycosylated IL-1RI and Il-1RAcP receptors and one cytokine [14]. Dynamics of the fully and partially glycosylated IL-1RI showed that the receptor often adopts a compact conformation upon glycosylation and remains in that state persistently [6]. Hence in this study, dynamics of the fully glycosylated IL-1RAcP was addressed first. RMSD plots showed that the fully glycosylated IL-1RAcP is noticeably flexible (Figure S.2.A). Visualization of the MD trajectories showed that the glycosylated IL-1RAcP remains consistently extended during the simulations time and does not adopt a compact conformation (Figure S.2.B-D).

\section{The non-signalling complex of compact IL-1RI with IL-1RAcP is highly stable}

The extended gIL-1RAcP forms two types of complexes with compact and extended gIL-1RI. The compact gIL-1RI-gIL-1RAcP type of complex must be the most populated IL-1RI assembly as the apo gIL-1RI is mainly in the compact state. Dynamics of the compact gIL-1RI-gIL-1RAcP complex was initially explored in six replicates of conventional MD simulations (Figures 2.A and S.3). All RMSD plots present a similar dynamical pattern for the complex. Where the accessory protein is the flexible unit of the complex and the IL-1RI is clearly more stable (Figure 2.A and S.3). Visualization of the MD trajectories shows that the compact gIL-1RI remains compact and connected to the gIL-1RAcP in all of the simulations (Figure 3). Calculation of the distance between the center of masses of DIII of gIL-1RI and DIII of gIL-1RAcP showed that the compact gIL-1RI-gIL-1RAcP complex presents the highest DIII-DIII distance with the highest fluctuations throughout the simulations (Figure S.4). Thus, the interactions between the compact gIL-1IRI and gIL-1RAcP are mainly through DII-DII of the two receptors (Figure 3) and they are weakened compared to the extended gIL-1RI-gIL-1RAcP complex (Figure 4). To ensure that the compact gIL-1RI-gIL-1RAcP is in fact stable, three replicates of aMD were carried out to enhance the conformational sampling of the system. Each aMD simulation was followed by another classical MD simulation to allow the system to regain its conventional dynamical pattern. RMSD plots present similar results to the classical MD simulations of the complex (Figure 2.B-C). Where the IL-1RI is the less flexible unit of the complex and the fluctuations are mainly raised by IL-1RAcP (Figure 2.B-C). Visualization of the simulation trajectories and distance calculations between DIII of the gIL-1RI and DIII of the gIL-1RAcP show that by undergoing the enhanced sampling, the complex becomes well-settled in the compact conformation (Figures S.3-S.4). The smallest distance values with the least fluctuations among the compact complex simulations were observed in the 
classical MD simulations that were carried out following the aMD simulations (Figures S.3). Approving that by increasing the simulation time, which is aimed to achieve with performing aMD, the compact gIL-1RIgIL-1RAcP complex becomes more stable. The compact gIL-1RI-gIL-1RAcP complex is a "non-signalling" complex as the compaction of the IL-1RI occludes its cytokine binding site (Figure 1.A). Thus the stability of the compact complex that is expected to be the most populated type of IL-1RI complex suggests a new down-regulation mechanism for the IL-1RI signalling that is different from the decoy receptor (IL-1RII) and binding of the receptor antagonist (IL-1ra) to IL-1RI that was reported before. This mechanism would be referred to as the non-signalling pathway here (Figure 1.A-B).

\section{The signalling complex is locked in the extended form by interconnected glycans}

It was suggested that the extended form of gIL-1RI also exist in a low population [6] and could form a complex with the gIL-1RAcP. The model of such complex was built (Figure 1.D) and its dynamical pattern was simulated here. RMSD plots of the extended glycosylated complex simulations show that similar to the compact complex, the overall flexibility raises from the IL-1RAcP dynamics while the IL-1RI is more stable (Figure 2.D). The distance between DIII-DIII of the two proteins is $~ 10 \AA$ smaller than all of the compact simulations and remains stable during the simulations time (Figure S.4). Visualization of the MD trajectories showed that the two oligosaccharide glycans from IL-1RI (attached to Asn216) and IL-1RAcP (attached to Asn279) "lock" the complex in the extended conformation by forming several persistent hydrogen bonds with the two proteins and within themselves (Figure 5.A). Detailed list of the persistent protein-glycan and glycan-glycan hydrogen bonds can be found in Tables S.2-S.3. This lock arrangement is formed in the way that the oligosaccharide attached to each subunit of the complex forms persistent hydrogen bonds with the opposite subunit of the complex (Figure 5.B-C). Such an arrangement would necessarily maintain the extended gIL-1RI-gIL-1RAcP complex in the extended form and does not allow the IL-1RI to adopt the compact conformation (Figure S.4). Furthermore, persistent hydrogen bonds between monosaccharides of each glycan contribute to the structural arrangement by maintaining the tertiary structure of the complex. While the gIL-1RI-gIL-1RAcP complex is locked in the extended form, cytokine binding site of the IL1RI extracellular domain will remain exposed and could interact with the ligands to form the signalling assembly (Figure 1.E). The strength of this hypothesis that the lock structural arrangement maintains the extended complex, can only be tested by removal of those two glycans that form this arrangement. As such experiment is surely feasible with molecular modelling, two sets of conventional MD simulations were performed on the models of the extended gIL-1RI-gIL-1RAcP complex with the glycans at Asn216(IL-1RI)Asn279(IL-1RAcP) and Asn216, Asn232(IL-1RI)-Asn279(IL-1RAcP) removed (Table S.1). The second model was considered to remove the oligosaccharide attached to Asn232 of the IL-1RI that is in the proximity of the lock arrangement in order to eliminate any plausible protein-glycan interaction in this region entirely. These two systems with the above-mentioned glycans removed from the complex will be referred to as the partially glycosylated complex. The dynamical pattern of the partially glycosylated complexes showed that unlike the fully glycosylated complex (Figure 1.D), the IL-1RI is the more flexible subunit of the complex while the IL1RAcP is stable (Figures 6.A-B and S.5). The flexibility of the IL-1RI in the partially glycosylated complexes is due to the compaction of the extended receptor or its close-by contact with the accessory protein (Figure 6.D-E). It was shown that the lack of locking interactions upon removal of the glycans lead to distability of the extended complex that mainly arises from the conformational changes of the IL-1RI. Visualization of the simulation trajectories and distance plots between the centers of masses of DIII of IL-1RI and DIII of IL-1RAcP shows that compared to the fully glycosylated complex, here the two DIIIs either dissociate from each other with $\sim 5 \AA$ increase in the distance values (Figure 6.C and 6.D) or they move close to each other with $\sim 3 \AA$ decrease in the distance values (Figure 6.C and 6.E). Both of these changes occurred within the first $10 \mathrm{~ns}$ of the trajectories and remained persistent throughout the simulations time. These observations showed that the lock arrangement does indeed maintain the extended gIL-1RI-gIL-1RAcP complex. One should note that according to the above-mentined observations, the role of this conformational arrangement is in maintaining the optimal distance between the two proteins and not solely in keeping them close to each other. And it is worth mentioning that although the time scale of the simulations here is very much limited compared to the currently available simulations in the biomolecular modelling field, the mechanism that was 
explained here is not an evolution of dynamics that is dependent on the simulation time. As the glycosidic linkages and formation of the complex are set apriori to the sampling. Thus, the time scale limitation of this work was overcome by performing several replicates of the extended system and by testing the proposed mechanism by removing the involved glycans and performing several control simulations.

\section{Discussion}

IL-1RI is arguably the most studied transmembrane receptor in the molecular machinery of immune responses upon several conditions such as asthma, arthritis, cancer, injury and infection $[1,3,4,56]$. The complexities that arise from binding of several agonist and antagonist ligands to IL-1RI extracellular domain $[1,13,14]$, functioning of homologous decoy $[2,18]$ and signalling transmembrane receptors that either compete with IL-1RI ligand binding in the extracellular matrix or trigger the overlapping inflammatory pathways in the intracellular matrix [57], makes this receptor's activation mechanism extremely challenging to fathom. Consequently, the in-silico drug design approaches that are extensively in use and have shown promising results in targeting other disease-related transmembrane receptors $[58,59]$ are limited in the case of IL-1RI. If one aims to narrow down the multi-dimensional functioning mechanism of IL-1RI to one fundamental step, it would be the formation of its functional assembly. In fact, several X-reay crystallographic studies attempted to address the formation of this assembly [13, 14, 20, 21]. However, as all the crystal structures of the functional assembly are obtained in full and by resolving only the extracellular domains of the IL-1RI and IL-1RAcP in an unglycosylated/underglycosylated form, the underlying molecular mechanism of its formation has remained unclear. And due to the extremely large size of this assembly that is consists of 828 amino acids and 11 oligosaccharides that each one consist of at least 5 monosaccharides, atomistic molecular modelling investigation of its formation mechanism is extremely challenging and computationally expensive. In this study, the entire molecular mechanism for the formation of glycosylated IL-1RI functional assembly is explained at the atomic level. Which showed that binding of the gIL-1RAcP to compact and extended conformations of gIL-1RI forms two types of stable non-signalling and signalling functional assembly respectively. The non-signalling assembly could act as a down regulating mechanism of IL-1RI functioning in the cell and the signalling assembly triggers the inflammatory pathways upon the IL- $1 \beta$ binding. Glycosylation is the structural basis of formation of both assemblies. By maintaining the gIL-1RI in the compact conformation that forms the non-signalling complex and by locking the singalling complex in the extended form. The locked assembly is maintained by specific arrangement of oligosaccharide glycans that are attached to Asn216, Asn232 of IL-1RI and Asn279 of IL-1RAcP. The arrangement is formed by several persistent hydrogen bonds between the oligosaccharides attached to each protein with the other protein in the complex and persistent hydrogen bonds within each oligosaccharide. However, there are two major drawbacks to this proposed mechanism. First is the lack of cell-membrane and transmembrane domains of IL-1RI and IL-1RAcP in the simulations and second is the limited time scale of the performed simulations. With fully appreciating these drawbacks, by referencing to several previous experimental and computational studies and performing advanced molecular modelling techniques here, it was attempted to overcome these drawbacks and provide comprehensive information to the community. MD simulations of the transmembrane receptors without embedding the transmembrane domains in the cell membrane has been carried out in several other studies of the same or similar receptors and they were successful to reproduce the experimental results if the system is relaxed properly $[6,34,60,61]$. And the time scale of the MD simulations is mainly dependent on the studied biological phenomenon. Here the glycosidic linkages and formation of each type of complex are set apriori to the sampling and the conformational transference between the two states was not required. The purpose of the MD simulations here is to observe the structural role of the attached glycans and their effect on the global dynamics of systems. This was achieved by performing several replicates of conventional MD simulations on each complex and by implementing accelerated MD to enhance the dynamics observed in the conventional MD (Table S.1). The mechanism of the lock arrangement in the extended complex was tested by removing the glycans proposed to regulated this arrangement and performing several control simulations (Table S.1 and Figure 6). Thus the mechanism proposed here is highly reliable based on the current experimental and computational available information of the system and could set the related drug-design attempts on a new path. Inhibition of the IL-1RI downstream signalling pathways at the genomic level could be experimentally 
achieved by mutation of the specific glycosylation sites; Asn216, Asn232 of IL-1RI and Asn279 of IL-1RAcP that their attached glycans form the lock arrangement. While promotion of the IL-1RI signalling could be feasible by introducing new glycosylation sites in the proximity of the lock arrangement that could reinforce the interactions. On the other hand, the computational drug-design approaches could focus on IL-1RI-IL1RAcP interactions rather than targeting the cytokine binding site. Monoclonal antibody or small peptide designs that block the IL-1RAcP binding to IL-1RI could severely suppress its signalling as it was shown before $[62,63]$. The above-mentioned suggestions for the biochemistry assays would be most effective if the mechanism presented here regarding the structural effects of glycans and the atomistic interactions they form is first experimentally validated. Expression and purification of IL-1RI and IL-1RAcP extracellular domains in the mammalian cells and analysis of the N-linked glycans by the mass spectroscopy method especially for the Asn279 of IL-1RAcP and Asn216 of IL-1RI that can participate in mutual glycan-protein interactions could be a reliable approach for this purpose.

\section{References}

1. Fields, J.K., Structural basis of IL-1 family cytokine signaling. Frontiers in immunology, 2019. 10 : p. 1412 .

2. Garlanda, C., et al., Decoys and regulatory "receptors" of the IL-1/Toll-like receptor superfamily. Frontiers in immunology, 2013.4 : p. 180.

3. Sims, J.E. and D.E. Smith, The IL-1 family: regulators of immunity. Nature Reviews Immunology, 2010. 10 (2): p. 89-102.

4. Krumm, B., Y. Xiang, and J. Deng, Structural biology of the IL-1 superfamily: Key cytokines in the regulation of immune and inflammatory responses. Protein Science, 2014. 23 (5): p. 526-538.

5. Bulek, K., et al., The essential role of single Ig IL-1 receptor-related molecule/Toll IL-1R8 in regulation of Th2 immune response. The Journal of Immunology, 2009. 182 (5): p. 2601-2609.

6. Azimzadeh Irani, M. and M.R. Ejtehadi, GAG positioning on IL-1RI; A mechanism regulated by dual effect of glycosylation.Glycobiology, 2019. 29 (11): p. 803-812.

7. Peters, V.A., J.J. Joesting, and G.G. Freund, IL-1 receptor 2 (IL-1R2) and its role in immune regulation. Brain, behavior, and immunity, 2013. 32 : p. 1-8.

8. Dinarello, C.A., Overview of the IL-1 family in innate inflammation and acquired immunity. Immunological reviews, 2018.281 (1): p. 8-27.

9. Garlanda, C., C.A. Dinarello, and A. Mantovani, The interleukin-1 family: back to the future. Immunity, 2013.39 (6): p. 1003-1018.

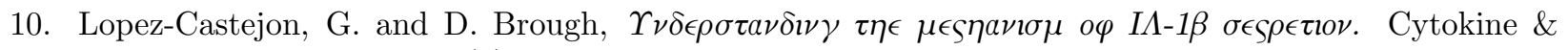
growth factor reviews, 2011.22 (4): p. 189-195.

11. Shaftel, S.S., W.S.T. Griffin, and M.K. O'Banion, The role of interleukin-1 in neuroinflammation and Alzheimer disease: an evolving perspective. Journal of neuroinflammation, 2008. 5 (1): p. 7.

12. Dinarello, C.A., Interleukin-1 Mediated Autoinflammation from Heart Disease to Cancer, in Textbook of Autoinflammation . 2019, Springer. p. 711-725.

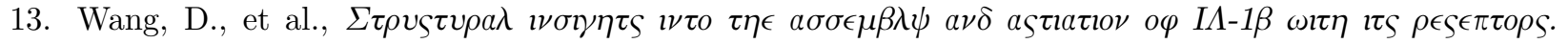
Nature immunology, 2010.11 (10): p. 905.

14. Thomas, C., J.F. Bazan, and K.C. Garcia, Structure of the activating IL-1 receptor signaling complex. Nature structural \& molecular biology, 2012. 19 (4): p. 455.

15. Yadav, B.S., et al., Protein modeling, molecular network and molecular dynamics study of newly sequenced interleukin-18 (IL-18) gene in Mus musculus. Journal of cellular physiology, 2019. 234 (8): p. 
14285-14295.

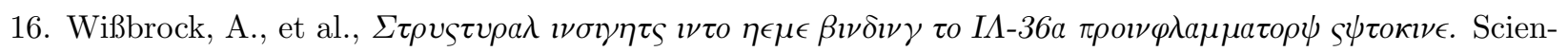
tific reports, 2019.9 (1): p. 1-14.

17. Ge, J., et al., Functional Relevance of Interleukin-1 Receptor Inter-domain Flexibility for Cytokine Binding and Signaling. Structure, 2019. 27 (8): p. 1296-1307. e5.

18. Colotta, F., et al., Interleukin-1 type II receptor: a decoy target for $I L-1$ that is regulated by $I L$-4. Science, 1993.261 (5120): p. 472-475.

19. Wald, D., et al., SIGIRR, a negative regulator of Toll-like receptor-interleukin 1 receptor signaling. Nature immunology, 2003.4 (9): p. 920-927.

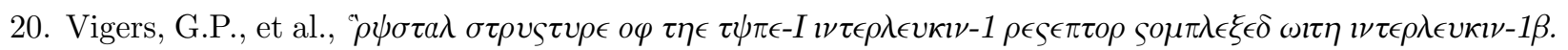
Nature, 1997.386 (6621): p. 190-194.

21. Vigers, G.P., et al., X-ray crystal structure of a small antagonist peptide bound to interleukin-1 receptor type 1. Journal of Biological Chemistry, 2000. 275 (47): p. 36927-36933.

22. Mariño, K., et al., A systematic approach to protein glycosylation analysis: a path through the maze. Nature chemical biology, 2010. 6 (10): p. 713.

23. Stanley, P., N. Taniguchi, and M. Aebi, N-glycans, inEssentials of Glycobiology [Internet]. 3rd edition . 2017, Cold Spring Harbor Laboratory Press.

24. Schulz, B.L., Beyond the sequon: sites of N-glycosylation.Rijeka, Croatia: INTECH, 2012: p. 21-39.

25. Taylor, M.E. and K. Drickamer, Introduction to glycobiology . 2011: Oxford university press.

26. Schwarz, F. and M. Aebi, Mechanisms and principles of N-linked protein glycosylation. Current opinion in structural biology, 2011.21 (5): p. 576-582.

27. Freeze, H.H. and M. Aebi, Altered glycan structures: the molecular basis of congenital disorders of glycosylation. Current opinion in structural biology, 2005. 15 (5): p. 490-498.

28. Shental-Bechor, D. and Y. Levy, Effect of glycosylation on protein folding: a close look at thermodynamic stabilization.Proceedings of the National Academy of Sciences, 2008. 105 (24): p. 8256-8261.

29. Slieker, L., T. Martensen, and M. Lane, Synthesis of epidermal growth factor receptor in human A431 cells. Glycosylation-dependent acquisition of ligand binding activity occurs post-translationally in the endoplasmic reticulum. Journal of Biological Chemistry, 1986.261 (32): p. 15233-15241.

30. Woods, R.J., Predicting the structures of glycans, glycoproteins, and their complexes. Chemical reviews, 2018.118 (17): p. 8005-8024.

31. Lee, H.S., Y. Qi, and W. Im, Effects of N-glycosylation on protein conformation and dynamics: Protein Data Bank analysis and molecular dynamics simulation study. Scientific reports, 2015.5 : p. 8926.

32. JOAO, H.C. and R.A. DWEK, Effects of glycosylation on protein structure and dynamics in ribonuclease $B$ and some of its individual glycoforms. European Journal of Biochemistry, 1993. 218 (1): p. 239-244.

33. Azimzadeh Irani, M., Correlation between experimentally indicated and atomistically simulated roles of EGFR N-glycosylation.Molecular Simulation, 2018. 44 (9): p. 743-748.

34. Azimzadeh Irani, M., S. Kannan, and C. Verma, Role of N-glycosylation in EGFR ectodomain ligand binding. Proteins: Structure, Function, and Bioinformatics, 2017. 85 (8): p. 1529-1549.

35. Kaszuba, K., et al., $<e m>N</ e m>$-Glycosylation as determinant of epidermal growth factor receptor conformation in membranes. Proceedings of the National Academy of Sciences, 2015.112 (14): p. 4334-4339. 
36. Arkhipov, A., et al., Membrane interaction of bound ligands contributes to the negative binding cooperativity of the EGF receptor.PLoS computational biology, 2014. 10 (7): p. e1003742-e1003742.

37. Yen, H.-Y., et al., Effect of sialylation on EGFR phosphorylation and resistance to tyrosine kinase inhibition.Proceedings of the National Academy of Sciences, 2015. 112 (22): p. 6955-6960.

38. Woods Group. (2005-XXXX) GLYCAM Web. Complex Carbohydrate Research Center, U.o.G., Athens, GA. (http://glycam.org), GLYCAM Web . (2005-2020)

39. D.A. Case, I.Y.B.-S., S.R. Brozell, D.S. Cerutti, T.E. Cheatham, III, V.W.D. Cruzeiro, T.A. Darden, R.E. Duke, D. Ghoreishi, M.K. Gilson, H. Gohlke, A.W. Goetz, D. Greene, R Harris, N. Homeyer, S. Izadi, A. Kovalenko, T. Kurtzman, T.S. Lee, S. LeGrand, P. Li, C. Lin, J. Liu, T. Luchko, R. Luo, D.J. Mermelstein, K.M. Merz, Y. Miao, G. Monard, C. Nguyen, H. Nguyen, I. Omelyan, A. Onufriev, F. Pan, R. Qi, D.R. Roe, A. Roitberg, C. Sagui, S. Schott-Verdugo, J. Shen, C.L. Simmerling, J. Smith, R. Salomon-Ferrer, J. Swails, R.C. Walker, J. Wang, H. Wei, R.M. Wolf, X. Wu, L. Xiao, D.M. York and P.A. Kollman (2018), AMBER 2018, University of California, San Francisco.

40. Pearlman, D.A., et al., AMBER, a package of computer programs for applying molecular mechanics, normal mode analysis, molecular dynamics and free energy calculations to simulate the structural and energetic properties of molecules. Computer Physics Communications, 1995. 91 (1): p. 1-41.

41. Maier, J.A., et al., ff14SB: Improving the Accuracy of Protein Side Chain and Backbone Parameters from ff99SB. Journal of Chemical Theory and Computation, 2015. 11 (8): p. 3696-3713.

42. Kirschner, K.N., et al., GLYCAM06: A generalizable biomolecular force field. Carbohydrates. Journal of Computational Chemistry, 2008. 29 (4): p. 622-655.

43. Leach, A.R., Molecular modelling : principles and applications . 2001, Harlow, England; New York: Prentice Hall.

44. Berendsen, H.J.C., et al., Molecular dynamics with coupling to an external bath. The Journal of Chemical Physics, 1984.81 (8): p. 3684-3690.

45. Pastor, R.W., B.R. Brooks, and A. Szabo, An analysis of the accuracy of Langevin and molecular dynamics algorithms. Molecular Physics, 1988. 65 (6): p. 1409-1419.

46. Loncharich, R.J., B.R. Brooks, and R.W. Pastor, Langevin dynamics of peptides: The frictional dependence of isomerization rates of $N$-acetylalanyl- $N$ '-methylamide. Biopolymers, 1992. 32 (5): p. 523-535.

47. Pierce, L.C.T., et al., Routine Access to Millisecond Time Scale Events with Accelerated Molecular Dynamics. Journal of Chemical Theory and Computation, 2012. 8 (9): p. 2997-3002.

48. Hamelberg, D., J. Mongan, and J.A. McCammon, Accelerated molecular dynamics: A promising and efficient simulation method for biomolecules. The Journal of Chemical Physics, 2004. 120 (24): p. 1191911929 .

49. Hamelberg, D., C.A.F.d. Oliveira, and J.A. McCammon, Sampling of slow diffusive conformational transitions with accelerated molecular dynamics. The Journal of Chemical Physics, 2007. 127 (15): p. 155102.

50. de Oliveira, C.A.F., et al., Large-scale conformational changes of Trypanosoma cruzi proline racemase predicted by accelerated molecular dynamics simulation. PLoS computational biology, 2011.7 (10): p. e1002178-e1002178.

51. Grant, B.J., A.A. Gorfe, and J.A. McCammon, Ras conformational switching: simulating nucleotidedependent conformational transitions with accelerated molecular dynamics. PLoS computational biology, 2009.5 (3): p. e1000325-e1000325. 
52. Humphrey, W., Dalke, A. and Schulten, K., "VMD - Visual Molecular Dynamics" J. Molec. Graphics 1996, 14.1, 33-38.

53. Roe, D.R. and T.E. Cheatham, PTRAJ and CPPTRAJ: Software for Processing and Analysis of Molecular Dynamics Trajectory Data. Journal of Chemical Theory and Computation, 2013. 9 (7): p. 3084-3095.

54. Durrant, J.D. and J.A. McCammon, HBonanza: a computer algorithm for molecular-dynamics-trajectory hydrogen-bond analysis.Journal of molecular graphics \& modelling, 2011. 31 : p. 5-9.

55. Schrodinger, L.L.C.T.P.m.g.s.V., no. 5 (2010): 0.

56. Gunther, S., et al., IL-1 Family Cytokines Use Distinct Molecular Mechanisms to Signal through Their Shared Co-receptor.Immunity, 2017. 47 (3): p. 510-523.e4.

57. Dinarello, C.A., Introduction to the interleukin-1 family of cytokines and receptors: Drivers of innate inflammation and acquired immunity. Immunological reviews, 2018. 281 (1): p. 5-7.

58. Sivasubramanian, A., et al., Structural Model of the mAb 806-EGFR Complex Using Computational Docking followed by Computational and Experimental Mutagenesis. Structure, 2006. 14 (3): p. 401-414.

59. Fuentes, G., et al., Synergy between trastuzumab and pertuzumab for human epidermal growth factor 2 (Her2) from colocalization: an in silicobased mechanism. Breast Cancer Research, 2011. 13 (3): p. R54.

60. Sanders, J.M., et al., Molecular determinants of epidermal growth factor binding: a molecular dynamics study. PloS one, 2013.8 (1).

61. Poger, D. and A.E. Mark, Activation of the epidermal growth factor receptor: a series of twists and turns. Biochemistry, 2014.53 (16): p. 2710-2721.

62. Quiniou, C., et al., Development of a Novel Noncompetitive Antagonist of IL-1 Receptor. The Journal of Immunology, 2008.180 (10): p. 6977-6987.

63. Lee, J.-H., et al., Type I IL-1 receptor (IL-1RI) as potential new therapeutic target for bronchial asthma. Mediators of inflammation, 2010. 2010 : p. 567351-567351.

\section{Acknowledgements}

We are grateful to the HPC of Sharif University of Technology for providing the computational resources of this study. Maryam is thankful to the Iranian National Elites Foundation for the "BMN-International" postdoctoral fellowship.

\section{Figure legends}

\section{Figure 1: Schematic presentation of the IL-1RI functional assemblies.}

IL-1RI functional assemblies mediate two non-signalling and signalling pathways through the IL-1RI compact and extended conformational states. Both compact and extended states co-exist in equilibrium while the former is the most populated conformation that is maintained by glycosylation. The non-signalling pathway works by interaction of the compact gIL-1RI with the gIL-1RAcP (A) to form the gIL-1RI-gIL-1RAcP secondary complex (B) . Extracellular domains of gIL-1RI and gIL-1RAcP are shown with magenta and marine cartoon/transparent surface respectively. Oligosaccharide glycans are shown with atom name sticks. Transmembrane domains and intracellular TIR domains are shown with yellow and dark yellow cartoons for both receptors. The membrane lipid bilayer is shown with grey cartoons. In the signalling pathway, the extended gIL-1RI that is expected to be the less populated conformation of the receptor, interacts with the gIL-1RAcP (C) and forms the gIL-1RI-gIL-1RAcP secondary complex(D). Agonist IL- $1 \beta$ binds to the secondary complex through its exposed binding site on the extended IL-1RI and forms the gIL-1RI-gIL1RAcP-IL-1 $\beta$ ternary complex (E) . C-E coloring is the same as the ones mentioned in A-B.

Figure 2. Average backbone RMSD plots of compact gIL-1RI-gIL-1RAcP (A-C) and extended gIL1RI-gIL-1RAcP complex(D) . Values were averaged over six replicates of the classical MD simulations. 
RMSD of the overall complex, IL-1RI and IL-1RAcP are presented with black, magenta and marine plots respectively.

Figure 3. Visualization of the glycosylated compact IL-1RI-IL-1RAcP complex from the classical MD simulations. Final structures of the simulations are presented in Figures A-F . Each structure was superimposed onto the first structure of its corresponding simulation. Extracellular domains of gIL-1RI and gIL-1RAcP are shown with magenta and marine surface respectively. Oligosaccharide glycans are shown with atom name sticks.

Figure 4. Distance between backbone atoms of the IL-1RI and IL-1RAcP interacting pairs are shown with blue and magenta plots respectively. Values are calculated from all replicate simulations of each system. The interacting pairs were reported in crystallographic structures of the IL-1RI-IL-1RAcP complex.

Figure 5. Extended IL-1RI-IL-1RAcP complex is locked in this state by several persistent hydrogen bonds formed between the extracellular domains of IL-1RI and IL-1RAcP with the other protein's glycans and the persistent hydrogen bonds within they glycans. Extracellular domains of IL-1RI and IL-1RAcP are shown with magenta and marine cartoon/transparent surface respectively. While both IL-1RI and IL-1RAcP are fully glycosylated, for the sake of simplicity, only the one oligosaccharide attached to IL-1RI and the one attached to IL-1RAcP that form the lock arrangement together are presented with atom name sticks here. The yellow and green colouring of the carbon atoms were used to present the oligosaccharides attached to IL-1RI and IL-1RAcP respectively. (A) The two oligosaccharides that lock the complex in the extended form are the ones attached to Asn216 of IL-1RI and Asn279 of IL-1RAcP. Upon binding of the extended IL-1RI and IL-1RAcP together, the glycans attached to each subunit of this dual-protein-construct forms persistent hydrogen bonds with the opposite subunit that acts as a lock to maintain the extended complex. The persistent hydrogen bonds within each oligosaccharide that support the tertiary structure of the locked extended complex are not shown here form simplicity. A detailed list of those hydrogen bonds can be found in Table S.3. (B) Visualization of some persistent hydrogen bonds formed between Asn216/Pro292(IL-1RI)Man5(IL-1RAcP attached glycan at Asn279) and (C) between Asn279/Thr273(IL-1RAcP)-Man5(IL-1RI attached glycan at Asn216) are presented with black dashed lines. The full list of these protein-glycan hydrogen bonds can be found in Table S.2.

Figure 6. Destabilizing effect of the "locking" glycans removal from the extended complex. Average backbone RMSD plots of the partially glycosylated extended IL-1RI-IL-1RAcP complex. Glycans were removed at Asn216 of IL-1RI and Asn279 of IL-1RAcP (A) and at Asn216 and Asn232 of IL-1RI and Asn279 of IL-1RAcP (B) . Values were averaged over three replicates of the classical MD simulations for each system. RMSD of the overall complex, IL-1RI and IL-1RAcP are presented with black, magenta and marine plots respectively.(C) Distance between center of masses of DIII of IL-1RI and DIII of IL-1RAcP was calculated for the partially glycosylated systems and it was compared with the values from the fully glycosylated extended complex. Values from the simulations of the partially glycosylated systems with the glycans removed at (Asn216 of IL-1RI and Asn279 of IL-1RAcP) and (at Asn216 and Asn232 of IL-1RI and Asn279 of IL-1RAcP) and the fully glycosylated complex are shown with violet, cyan and magenta plots respectively. (D-E) Visualization of the destabilizing effect of the removal of the glycans that are involved in maintaining the IL-1RI-IL-1RAcP complex in the extended form. Elimination of these glycans will either result in compaction of the IL-1RI (D) or a close-by contact between DIII-DIII of the two receptors that disturbs the optimal distance. 

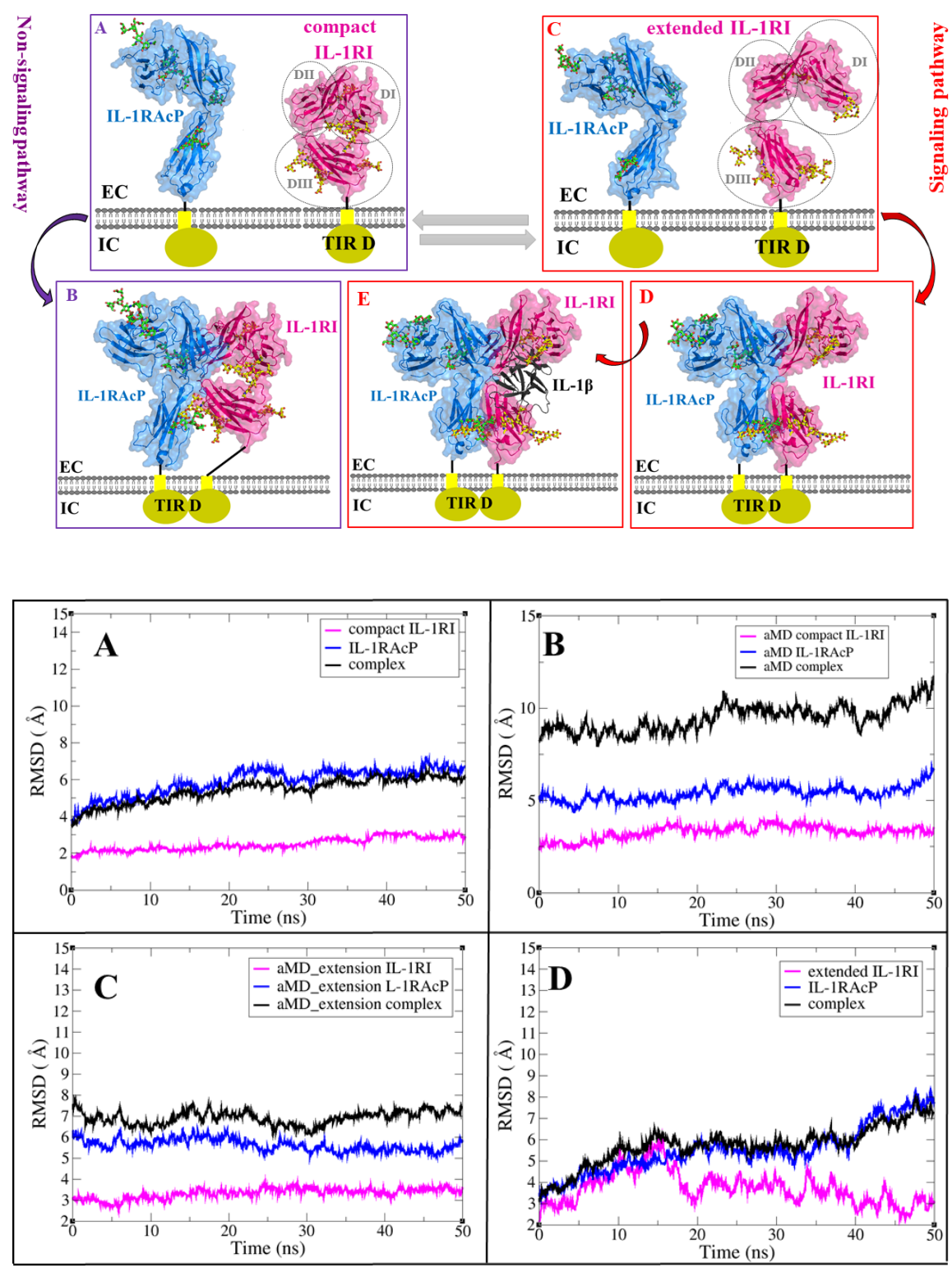

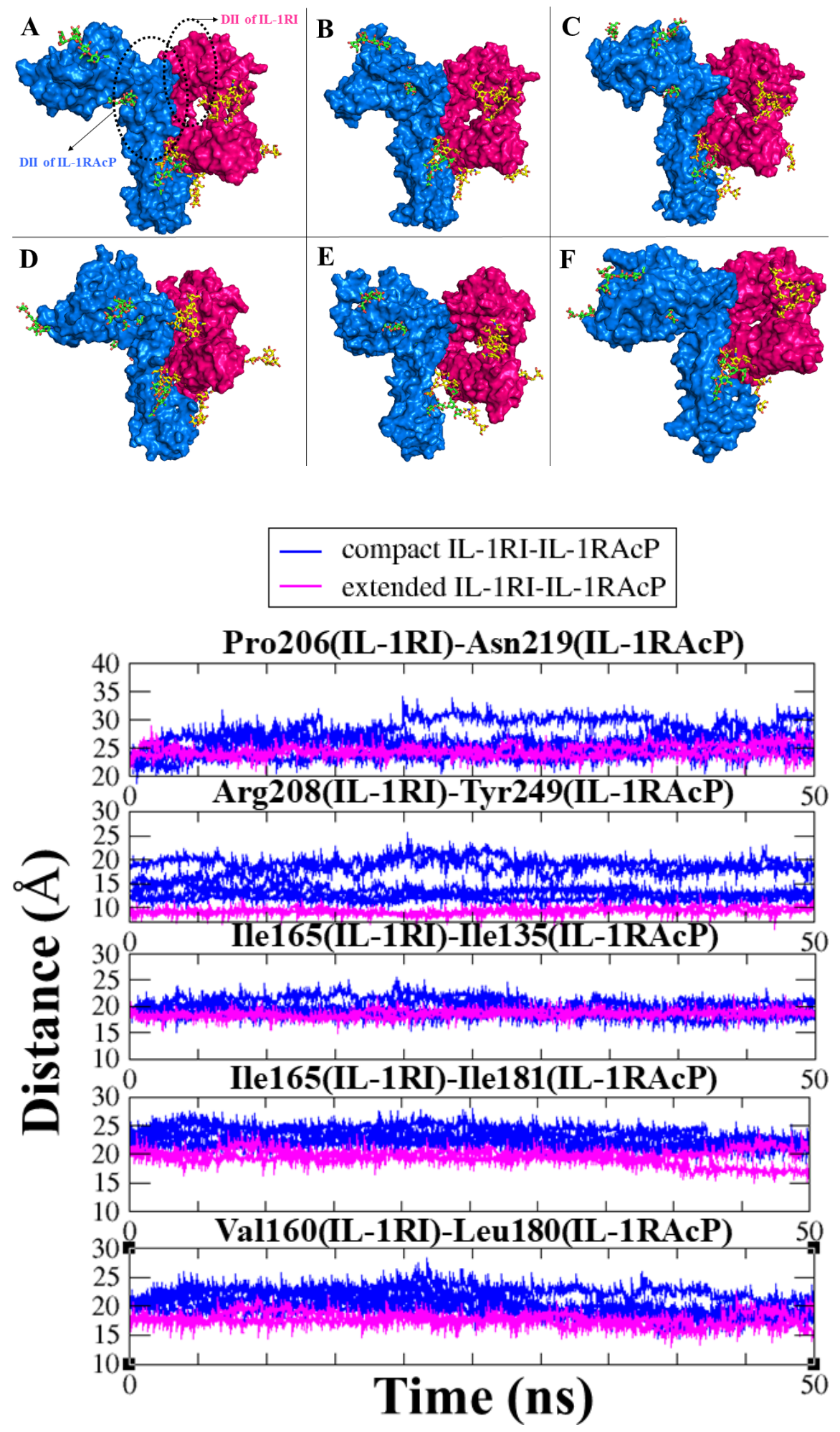

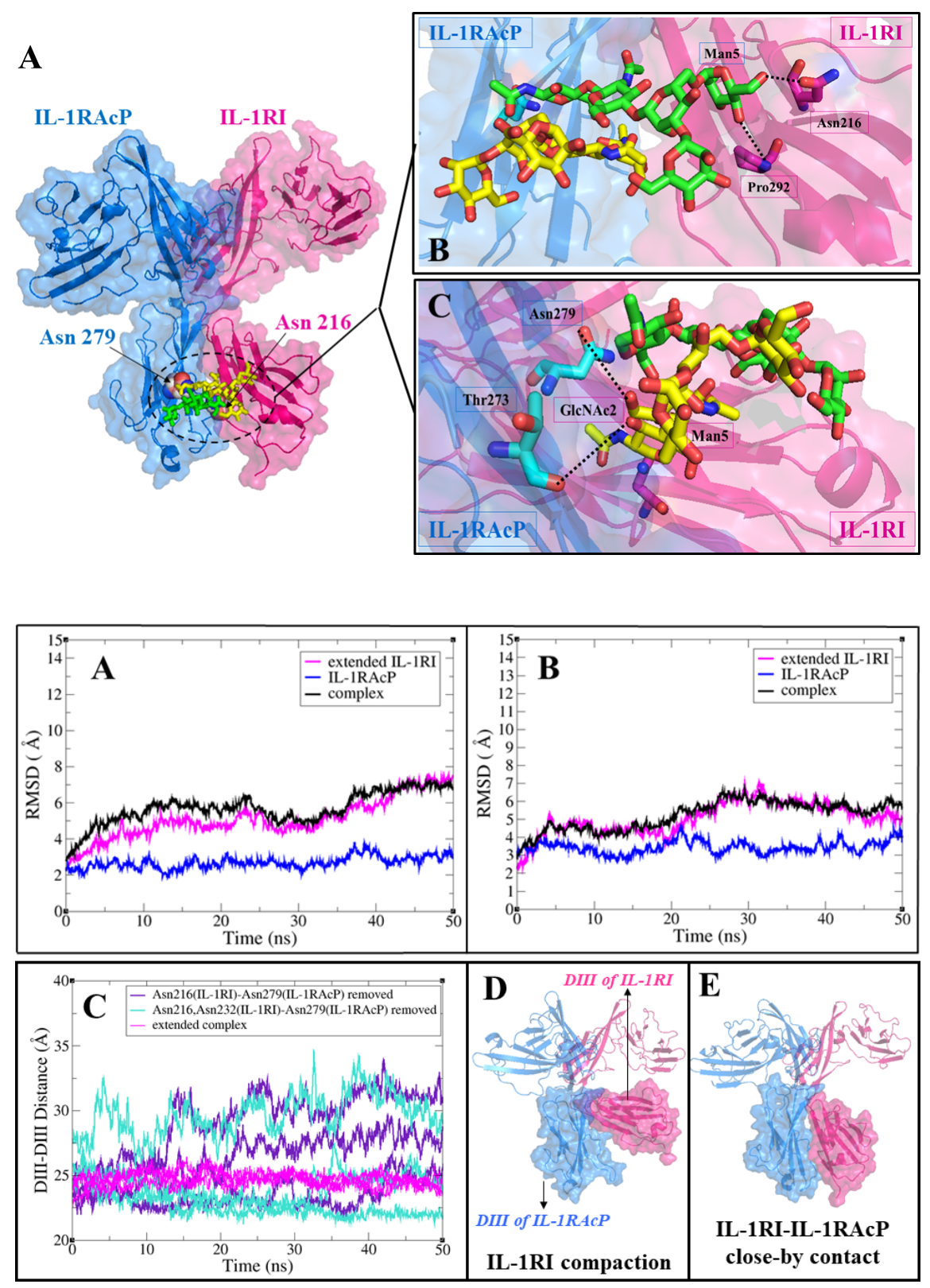